\title{
Glucagon secretion after metabolic surgery in diabetic rodents
}

\author{
Hans Eickhoff',2, Teresa Louro ${ }^{2,3}$, Paulo Matafome ${ }^{2,3}$, Raquel Seiça ${ }^{2,3}$ and \\ Francisco Castro e Sousa ${ }^{4,5}$ \\ ${ }^{1}$ Obesity Center, Hospital de Santiago, EN 10, km 37, 2900-722 Setubal, Portugal \\ ${ }^{2}$ Faculty of Medicine, Institutes of Physiology and ${ }^{3}$ Biomedical Imaging and Life Sciences (IBILI), \\ University of Coimbra, Polo III, Azinhaga de Santa Comba, Celas, 3000-548 Coimbra, Portugal \\ ${ }^{4}$ Department of Surgery A, University Hospital of Coimbra, Rua Fonseca Pinto, 3000-075 Coimbra, Portugal \\ ${ }^{5}$ Faculty of Medicine, University of Coimbra, Rua Larga, 3004-504 Coimbra, Portugal
}

\author{
Correspondence \\ should be addressed \\ to H Eickhoff \\ Email \\ h.c.a.e@sapo.pt
}

\begin{abstract}
Excessive or inadequate glucagon secretion promoting hepatic gluconeogenesis and glycogenolysis is believed to contribute to hyperglycemia in patients with type 2 diabetes. Currently, metabolic surgery is an accepted treatment for obese patients with type 2 diabetes and has been shown to improve glycemic control in Goto-Kakizaki (GK) rats, a lean animal model for type 2 diabetes. However, the effects of surgery on glucagon secretion are not yet well established. In this study, we randomly assigned forty 12- to 14-week-old GK rats to four groups: control group (GKC), sham surgery (GKSS), sleeve gastrectomy (GKSG), and gastric bypass (GKGB). Ten age-matched Wistar rats served as a non-diabetic control group (WIC). Glycemic control was assessed before and 4 weeks after surgery. Fasting- and mixed-meal-induced plasma levels of insulin and glucagon were measured. Overall glycemic control improved in GKSG and GKGB rats. Fasting insulin levels in WIC rats were similar to those for GKC or GKSS rats. Fasting glucagon levels were highest in GKGB rats. Whereas WIC, GKC, and GKSS rats showed similar glucagon levels, without any significant meal-induced variation, a significant rise occurred in GKSG and GKGB rats, 30 min after a mixed meal, which was maintained at 60 min. Both GKSG and GKGB rats showed an elevated glucagon:insulin ratio at $60 \mathrm{~min}$ in comparison with all other groups. Surprisingly, the augmented post-procedural glucagon secretion was accompanied by an improved overall glucose metabolism in GKSG and GKGB rats. Understanding the role of glucagon in the pathophysiology of type 2 diabetes requires further research.
\end{abstract}

\section{Key Words}

- metabolic surgery

- Goto-Kakizaki rats

- glucagon

- insulin

\section{Introduction}

Pathophysiology of type 2 diabetes is commonly characterized by a relative insufficiency of pancreatic $\beta$-cells secreting insulin in response to a given blood glucose level to maintain normoglycemia in the presence of an increased hepatic and peripheral insulin resistance. Lipotoxicity, glucotoxicity, and islet amloid polypeptide (IAPP) deposition have been implicated in progressive $\beta$-cell failure (DeFronzo 2009). Changes in incretin levels and action such as resistance to glucose-dependent insulinotropic peptide (GIP) and reduced glucagon-like peptide 1 (GLP1) secretion seem to play an additional role in the development of type 2 diabetes (Holst et al. 2009). Excessive or inadequate glucagon secretion promoting hepatic gluconeogenesis and glycogenolysis probably also contributes to impaired glucose tolerance in diabetic patients (Gastaldelli et al. 2000).

Modification of the gastrointestinal tract by bariatric or metabolic surgery currently represents an accepted 
treatment for type 2 diabetes in obese patients (Dixon et al. 2011), with overall remission rates of $78.1 \%$ as reported in a recent meta-analysis (Buchwald et al. 2009). Favorable effects of surgery on glucose metabolism have also been observed in non-severely or morbidly obese patients (BMI $<35 \mathrm{~kg} / \mathrm{m}^{2}$ ) with type 2 diabetes (Scopinaro et al. 2011); yet, surgical treatment in this patient group is still considered investigational and level 1 evidence is missing (Shukla et al. 2012). Research on incretins and other gut hormones and their modification through surgical treatment revealed their importance in diabetes remission (Thaler \& Cummings 2009, Papamargaritis et al. 2013). However, little is known about the effect of surgery on glucagon metabolism and its influence on outcome regarding remission of diabetes.

Goto-Kakizaki (GK) rats have been studied extensively as a model for type 2 diabetes in non-obese humans, and display similar features in comparison with the human pancreatic phenotype in patients with type 2 diabetes (Seiça et al. 2003, Portha et al. 2009). Thus, we studied the effect of common bariatric procedures such as sleeve gastrectomy and gastric bypass on glucose metabolism, insulin sensitivity, and pancreatic hormone secretion in this rodent model.

\section{Materials and methods}

\section{Study protocol}

Male GK rats aged 12-14 weeks and age-matched Wistar rats were obtained from the Laboratory for Experimental Research, Faculty of Medicine of the University of Coimbra, Coimbra, Portugal. The animals were maintained in a controlled environment with day-night cycles of $12 \mathrm{~h}$, with a temperature of $22-24^{\circ} \mathrm{C}$ and a relative humidity of $50-60 \%$. Ad libitum access to water and standard rat chow (Charles River, SAFE, L'Arbresle, France) was provided. The operated animals had additional access to a mixed liquid diet during the first post-operative days (Forticare, Nutricia, Danone Group, Paris, France).

The study protocol regarding the use of laboratory animals was developed taking into account the ARRIVE guidelines (Kilkenny et al. 2010) and according to the procedures approved by the Institutional Animal Care and Use Committee. After completing the study, animals were killed by cervical displacement.

Forty non-obese diabetic GK rats were randomly assigned to the control (GKC), sham surgery (GKSS), sleeve gastrectomy (GKSG), and gastric bypass (GKGB). Ten non-diabetic Wistar rats served as an additional control group (WIC). Before surgery or entering the control group, weight was recorded and venous blood was obtained from the tail vein after an overnight fast in order to determine fasting blood glucose and HbA1clevels. An oral glucose tolerance test (OGTT) was performed with an oral glucose load of $1.75 \mathrm{~g} / \mathrm{kg}$ body weight and blood glucose reassessed after 30,60 , and $120 \mathrm{~min}$.

The animals assigned to surgery (GKSS, GKSG, and GKGB) were operated upon after being anesthetized using i.m. ketamine $(75 \mathrm{mg} / \mathrm{kg}$ bodyweight) and chlorpromazine ( $3 \mathrm{mg} / \mathrm{kg}$ bodyweight). After preparing the abdomen, a midline incision was made and entered the abdominal cavity. Surgery was performed as described below and according to the randomization of animals. After completing surgery, the abdomen was rinsed with sterile saline and meloxicam $(1 \mathrm{mg} / \mathrm{kg}$ bodyweight, Boehringer Ingelheim $\mathrm{GmbH}$, Ingelheim, Germany) was instilled in the peritoneal cavity for post-operative analgesia. In all animals, the abdominal wall was closed by continuous mass closure with a $3 / 0$ short-term absorbable suture of braided and coated polyglycolic acid (Safil Quick, B Braun, Aesculap AG, Tuttlingen, Germany). Post-operative care included s.c. analgesia with meloxicam $(1 \mathrm{mg} / \mathrm{kg}$ bodyweight) and oral antibiotics (doxycycline, $5 \mathrm{mg} / \mathrm{kg}$ bodyweight, Pfizer Inc., New York, USA) mixed with drinking water for 5 days. The animals dying from postoperative complications $(n=12)$ were substituted to maintain the original number of animals in groups.

At 4 weeks, body weight was reassessed and blood samples without prior fasting were drawn from the caudal vein for the measurement of random glycemia. After an overnight fast, analysis of blood samples was repeated for fasting glycemia and HbA1c. An OGTT was performed as described earlier. Three to four days later, for the determination of plasma insulin and glucagon blood samples were obtained by intra-cardiac puncture in anesthetized animals, before and after they were orally gavaged with $3 \mathrm{cc}$ of a mixed liquid diet (Forticare) supplied by Nutricia, Danone Group. The animals were killed afterwards as described earlier.

\section{Surgical technique}

Surgery was performed as described previously (Eickhoff et al. 2014). Briefly, we mobilized the stomach and made a $3 \mathrm{~cm}$ incision in anterior gastric wall in animals assigned to sham surgery. To resemble more complex surgical techniques, the gastric incision was left open for 20 min before closing by a continuous extra-mucosal technique using a $4 / 0$ mid-term absorbable synthetic

Published by Bioscientifica Ltd. 
monofilament suture made of glyconate (Monosyn, B Braun, Aesculap AG).

We performed sleeve gastrectomy by dissecting the greater curvature including the lower part of the stomach and the aglandular forestomach with ligation of the short gastric vessels, similar to a technique described elsewhere (de Bona Castelan et al. 2007). After sectioning the stomach over a bulldog clamp to fashion the gastric sleeve, we removed the operative specimen, including most of the forestomach. The gastric wound was closed by a continuous invaginating extra-mucosal suture with a $4 / 0$ mid-term absorbable synthetic glyconate monofilament thread as described earlier.

Gastric bypass surgery was carried out in an analogous manner to that in humans and as described for rats (Xu et al. 2002, Meguid et al. 2004). The mid portion of the stomach of animals allocated to gastric bypass was freed from adhesions on the greater and lesser curvature. A horizontal section of the stomach was performed leaving only a small rim of glandular stomach on the proximal part. The distal stomach was closed using a $4 / 0$ mid-term absorbable monofilament suture (Monosyn, B Braun, Aesculap AG) in an invaginating extra-mucosa technique. On the lesser curvature, the proximal stomach was sutured in a similar fashion, leaving a small opening close to the greater curvature to perform the gastro-jejunal anastomosis. We identified the angle of Treitz and measured $20 \mathrm{~cm}$ on the proximal jejunum. The jejunal loop was brought up to the proximal stomach and a $2 \mathrm{~cm}$ incision was made on the antimesenteric border of the bowel wall. The gastrojejunal anastomosis was performed in an end-to-side fashion by an extramucosal continuous suture using a 5/0 mid-term absorbable synthetic monofilament suture made of glyconate (Monosyn, B Braun, Aesculap AG). No mesenteric defects were closed.

\section{Glucose metabolism and hormones}

Blood sugar was evaluated by a procedure based on glucose-oxidase reaction using a commercially available glucometer with appropriate test strips (Glucometer Elite, Bayer SA). HbA1c was measured using a Siemens DCA $2000+$ Analyzer (Siemens AG, Munich, Germany). Glucagon was analyzed by a competitive ELISA (Wako Pure Chemical Industries Ltd, Osaka, Japan), using specific antibodies that bind to sample and biotinylated tracer respectively. A 96-well microtiter plate coated directly with the antibody bound to sample or tracer was subsequently submitted to the reaction of captured tracer with HRP-conjugated streptavidin, which produces a colored product after addition of a substrate.

Plasma insulin was analyzed in a similar fashion using a non-competitive solid-phase two-site enzyme immunoassay based on the sandwich technique (Mercodia AB, Uppsala, Sweden) in which insulin in the sample reacts to specific antibodies bound to the microtiter plate and peroxidase-conjugated antibodies in a solution producing a colored solution together with a substrate. The duplicate samples of hormones were measured using a microplate reader with Gen5 Software (Synergy HT, Biotek, Winooski, VT, USA).

Insulin sensitivity was assessed by a mathematical model (quantitative insulin sensitivity check index) calculating the reciprocal index of the sum of logarithms for fasting plasma glucose $(\mathrm{mg} / \mathrm{dl})$ and fasting plasma insulin $(\mu \mathrm{U} / \mathrm{ml})>\left(1 /\left(\log \left(\mathrm{G}_{0}\right)+\log \left(\mathrm{I}_{0}\right)\right)\right)$ as described elsewhere (Katz et al. 2000, Muniyappa et al. 2009). The area under the curve (AUC) was calculated for postprandial insulin and glucagon secretion using the trapezoidal method.

\section{Statistical analysis}

All data were described as mean \pm s.E.M. with respect to the number of experiments described. The Shapiro-Wilk $U$ test was used to assess whether the samples were extracted from a variable that follows a normal distribution. As some variables were not normally distributed, the non-parametric Mann-Whitney $U$ test was used to reject or keep the null hypothesis that there were no differences in means of groups $\left(\mathrm{H}_{0}: \mu_{1}=\mu_{2}\right)$. To reduce type 1 errors induced by repeated Mann-Whitney tests, the KruskalWallis test and post hoc analysis including multiple pairwise comparisons using the Steel-Dwass-CritchlowFligner procedure was employed to describe the differences between more than two groups with respect to the same variable or repeated measures of a variable in the same group. The differences were considered statistically significant at a level of $95 \%(P<0.05)$.

\section{Results}

At the beginning and at the end of the observation period, Wistar rats were heavier than GK rats, independent of allocated treatment. Fasting glycemia at baseline was similar in all groups $(78 \pm 2.1-106 \pm 13.0 \mathrm{mg} / \mathrm{dl})$, except for GKSS, but $2 \mathrm{~h}$ after an oral glucose load, blood glucose was significantly worse in GK rats (all groups) in comparison with WIC $(125 \pm 11.7-169 \pm 11.9$ vs $84.1 \pm 3.1, P<0.01)$. At the end of the observation period, WIC presented

Published by Bioscientifica Ltd 
with a significantly lower fasting glycemia $(72 \pm 3.9 \mathrm{mg} / \mathrm{dl})$ than GK rats in all groups $(106 \pm 4.9-136 \pm 9.2 \mathrm{mg} / \mathrm{dl})$. At the same time, random glycemia was also lower in WIC $(87 \pm 2.4 \mathrm{mg} / \mathrm{dl})$ in comparison with $\mathrm{GK}$ rats $(P<0.02)$, except for GKSG $(106 \pm 12.3 \mathrm{mg} / \mathrm{dl})$, and blood glucose levels after an OGTT were significantly higher in GK rats (all groups, $P<0.05$ ) (Fig. 1). Similarly, overall glycemic control assessed by HbA1c levels was better in WIC in comparison with GK at the beginning and at the end of the observation period. However, GK rats submitted
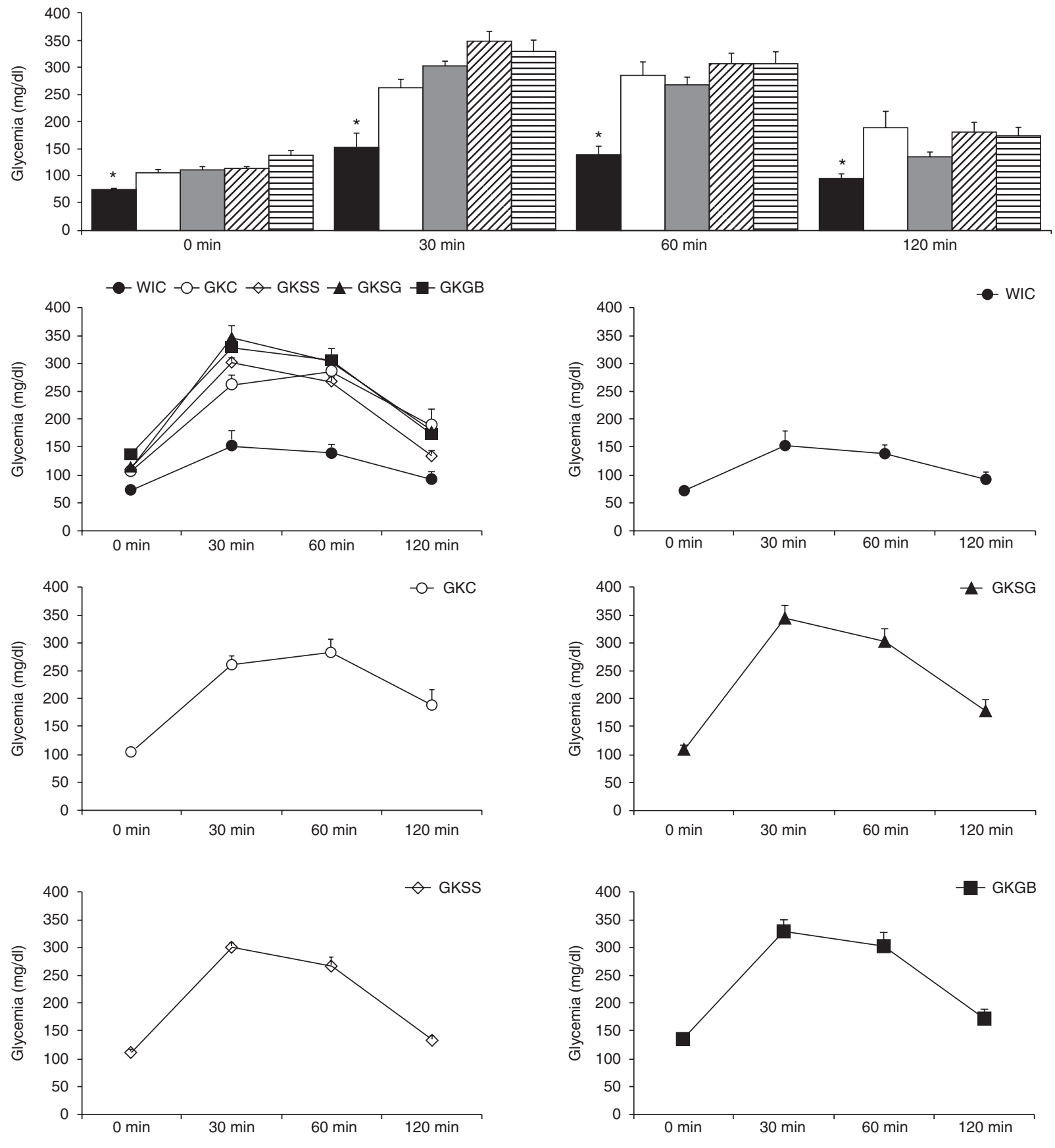

Figure 1

Oral glucose tolerance test. WIC, Wistar rats - control; GKC, Goto-Kakizaki rats - control; GKSS, Goto-Kakizaki rats - sham surgery; GKSG,

Goto-Kakizaki rats - sleeve gastrectomy; GKGB, Goto-Kakizaki rats - gastric

bypass. Kruskal-Wallis test with post hoc analysis: $* P<0.05$ in comparison with GKC, GKSS, GKSG, and GKGB. 
to sleeve gastrectomy or gastric bypass improved their HbA1c concentration significantly in comparison with pre-operative values $(P<0.005$ and $P<0.05$ respectively) and in comparison with GKC and GKSS rats (Table 1).

Fasting plasma insulin levels were similar in Wistar and GK rats, whether submitted to surgery or not. Mealinduced plasma insulin concentrations at $20 \mathrm{~min}$ were significantly higher in GK rats (all groups) in comparison with WIC $(P<0.05)$. At $30 \mathrm{~min}$, plasma insulin was highest in GKSG rats $(17.48 \pm 0.75 \mu \mathrm{g} / \mathrm{l} ; P<0.05$ in comparison with all other groups) and lowest in GKGB rats $(9.21 \pm 0.39 \mu \mathrm{g} / \mathrm{l})$. Similar results were obtained at $40 \mathrm{~min}$. However, at $60 \mathrm{~min}$ both GKSG and GKGB rats presented with lower plasma insulin than other groups $(5.95 \pm 0.28$ and $4.77 \pm 0.63 \mu \mathrm{g} / \mathrm{l} ; P<0.05)$. Time series revealed a significant rise in comparison with baseline at $20 \mathrm{~min}$ in all groups $(P<0.05)$, but whereas GKC and GKSS rats maintained similar values throughout all following time points, a significant reduction in plasma insulin concentration was observed in WIC, GKSG, and GKGB rats at $60 \mathrm{~min}$ in comparison with the previous time point $(P<0.05)$ (Fig. 2). Total insulin secretion during the first $60 \mathrm{~min}$ after a liquid mixed-meal expressed as AUC was highest in GKC $(614 \pm 18.3 \mathrm{~h} \times \mu \mathrm{g} / \mathrm{l} ; P<0.05$ in comparison with GKSS, and GKGB) and lowest in GKGB rats $(396 \pm 15.3 \mathrm{~h} \times \mu \mathrm{g} / \mathrm{l} ; \quad P<0.05$ in comparison with all other GK). The differences between Wistar and GK rats in all groups were not statistically significant (Table 1).

Insulin sensitivity was assessed using the quantitative insulin sensitivity check index as described earlier. GK rats in the control group and submitted to sham surgery presented with a lower insulin sensitivity $\left(0.2601 \pm 2.5^{-3}\right.$ and $0.25 \pm 5.3^{-3}$ respectively) than Wistar rats $(0.284 \pm$ $\left.1.9^{-3} ; P<0.05\right)$. GKSG and GKGB had slightly improved indices in comparison with GKC and GKSS rats, and no significant differences in comparison with Wistar rats were observed (Table 1).

GK rats submitted to gastric bypass presented with higher fasting plasma glucagon concentrations than all other groups $(405.86 \pm 30.14 \mathrm{pg} / \mathrm{ml} ; P<0.05$ in comparison with GKC and GKSG) and maintained this level at 10 and $20 \mathrm{~min}$ after the test meal. However, at $30 \mathrm{~min}$, plasma glucagon levels increased significantly in rats submitted to sleeve gastrectomy $(676.04 \pm 18.01 \mathrm{pg} / \mathrm{ml})$, as well as, to a lesser extent, in rats submitted to gastric bypass $(652.37 \pm 39.69 \mathrm{pg} / \mathrm{ml})$. Hormone levels remained stable at a high level in both groups up to $60 \mathrm{~min}(P<0.05$

Table 1 Values at baseline and at the end of observation period for weight, blood glucose, HbA1c, insulin sensitivity, plasma insulin, and glucagon. All values presented with S.E.M.

\begin{tabular}{|c|c|c|c|c|c|}
\hline & WIC & GKC & GKSS & GKSG & GKGB \\
\hline \multicolumn{6}{|l|}{ Weight (g) } \\
\hline Baseline & $328 \pm 11.9^{a}$ & $256 \pm 9.0$ & $270 \pm 13.4$ & $277 \pm 7.3$ & $284 \pm 9.2$ \\
\hline 4 weeks & $388 \pm 9.1^{\neq \mathrm{b}}$ & $308 \pm 8.0^{\ddagger}$ & $330 \pm 11.5^{\dagger}$ & $296 \pm 17.8$ & $303 \pm 12.5$ \\
\hline \multicolumn{6}{|l|}{ Fasting glycemia (mg/dl) } \\
\hline Baseline & $78 \pm 2.1^{c}$ & $102 \pm 11.3$ & $110 \pm 10.9$ & $97 \pm 8.3$ & $106 \pm 13.0$ \\
\hline 4 weeks & $72 \pm 3.9^{d}$ & $106 \pm 4.9$ & $111 \pm 5.7$ & $112 \pm 5.3 *$ & $136 \pm 9.2 *$ \\
\hline \multicolumn{6}{|l|}{2 h OGTT $(\mathrm{mg} / \mathrm{dl})$} \\
\hline Baseline & $84 \pm 3.1^{d}$ & $169 \pm 11.9$ & $155 \pm 12.4$ & $138 \pm 9.6$ & $125 \pm 11.7$ \\
\hline 4 weeks & $93 \pm 10.8^{b}$ & $190 \pm 29.3$ & $135 \pm 8.0$ & $179 \pm 18.5$ & $173 \pm 16.1$ * \\
\hline \multicolumn{6}{|l|}{ Random glycemia (mg/dl) } \\
\hline 4 weeks & $87 \pm 2.4^{\mathrm{e}}$ & $169 \pm 30.4$ & $153 \pm 20.5$ & $106 \pm 12.3$ & $129 \pm 13.2$ \\
\hline \multicolumn{6}{|l|}{ HbA1c (\%) } \\
\hline Baseline & $3.5 \pm 0.1^{d}$ & $4.8 \pm 0.3$ & $4.8 \pm 0.3$ & $5.1 \pm 0.3$ & $5.0 \pm 0.3$ \\
\hline 4 weeks & $3.3 \pm 0.1^{b}$ & $5.0 \pm 0.2$ & $4.6 \pm 0.2$ & $3.8 \pm 0.1^{\neq f}$ & $4.0 \pm 0.2 * 9$ \\
\hline \multicolumn{6}{|l|}{ Insulin sensitivity (QUICKI) } \\
\hline 4 weeks & $0.284 \pm 1.9^{-3 f}$ & $0.2601 \pm 2.5^{-3}$ & $0.25 \pm 5.3^{-3}$ & $0.2678 \pm 6.4^{-3}$ & $0.2659 \pm 4.4^{-3}$ \\
\hline \multicolumn{6}{|l|}{ Insulin AUC $(\mathrm{h} \times \mu \mathrm{g} / \mathrm{l})$} \\
\hline 4 weeks & $532 \pm 29.3$ & $614 \pm 18.3^{c}$ & $521 \pm 12.6$ & $592 \pm 19.6^{h}$ & $396 \pm 15.3^{i}$ \\
\hline \multicolumn{6}{|l|}{ Glucagon AUC $(\mathrm{h} \times \mathrm{ng} / \mathrm{ml})$} \\
\hline 4 weeks & $22.9 \pm 2.7$ & $15.0 \pm 3.0$ & $18.1 \pm 2.0$ & $29.9 \pm 0.8^{f}$ & $31.5 \pm 1.7^{f}$ \\
\hline
\end{tabular}

WIC, Wistar rats - control; GKC, Goto-Kakizaki rats - control; GKSS, Goto-Kakizaki rats - sham surgery; GKSG, Goto-Kakizaki rats - sleeve gastrectomy; GKGB, Goto-Kakizaki rats - gastric bypass; OGTT, oral glucose tolerance test; AUC, area under the curve; QUICKI, quantitative insulin sensitivity check index. The Mann-Whitney $U$ test was used to assess differences between baseline and values at the end of the observation period ( $P<0.05$, $\uparrow P<0.01$, and $¥ P<0.005$ ). The Kruskal-Wallis test was used to assess intergroup differences ( ${ }^{\mathrm{a}} P<0.05$ in comparison with GKC, GKSG, and GKGB; ${ }^{b} P<0.05$ in comparison with GKC, GKSS, GKSG, and GKGB; ${ }^{C} P<0.05$ in comparison with GKSS; ${ }^{d} P \leq 0.01$ in comparison with GKC, GKSS, GKSG, and GKGB; e $P<0.02$ in comparison with GKC, GKSS, and GKGB; ${ }^{f} P<0.05$ in comparison with GKC, and GKSS; ${ }^{9} P<0.05$ in comparison with GKC; ${ }^{\mathrm{h}} P<0.05$ in comparison with GKGB; and ${ }^{\mathrm{i}} P<0.05$ in comparison with GKC, GKSS, and GKSG).

http://joe.endocrinology-journals.org DOI: 10.1530/JOE-14-0445
() 2014 Society for Endocrinology Printed in Great Britain
Published by Bioscientifica Ltd. 

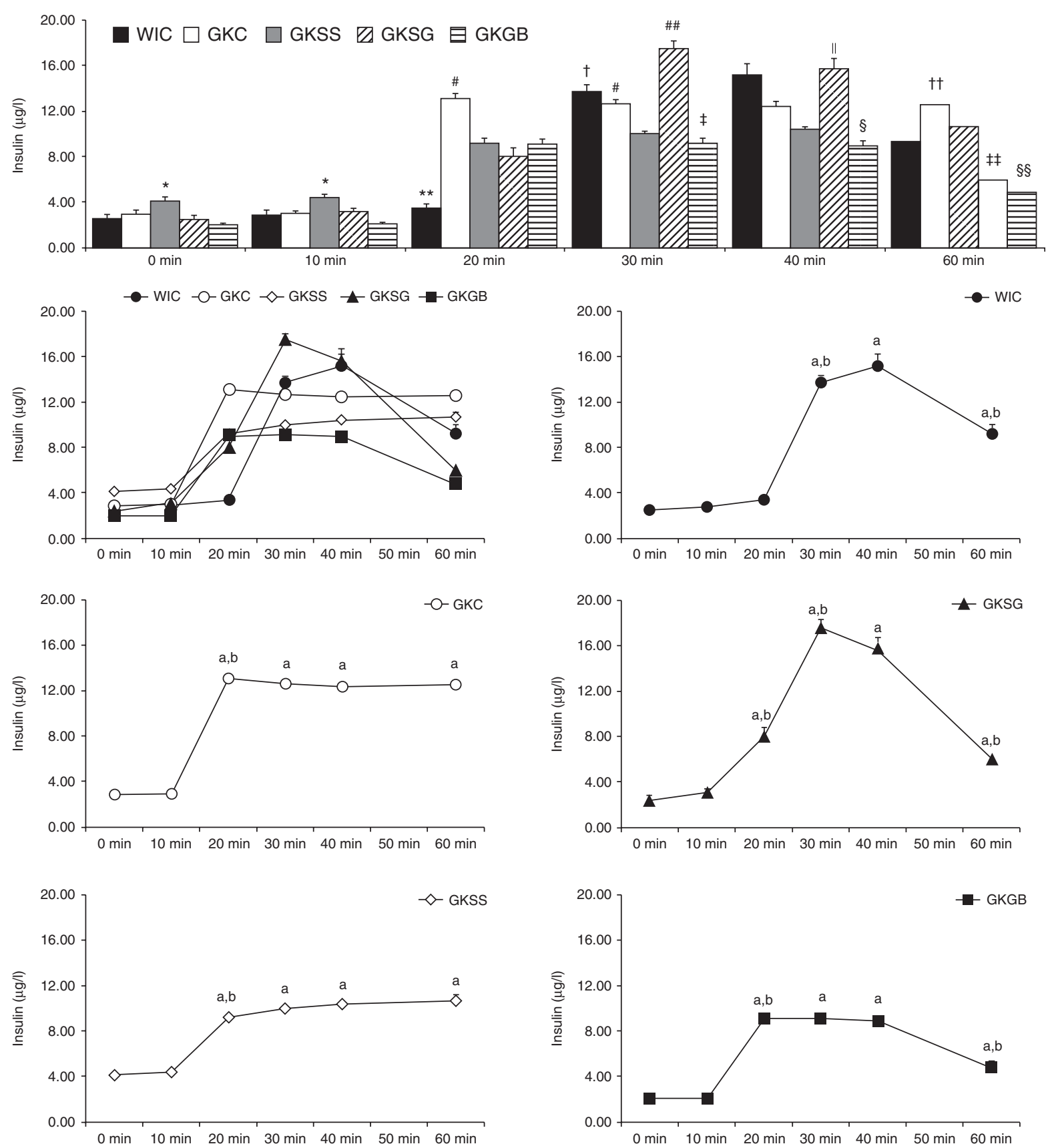

\section{Figure 2}

Insulin level - meal-induced response. WIC, Wistar rats - control; GKC, Goto-Kakizaki rats - control; GKSS, Goto-Kakizaki rats - sham surgery; GKSG, Goto-Kakizaki rats - sleeve gastrectomy; GKGB, Goto-Kakizaki rats - gastric bypass. Kruskal-Wallis test with post hoc analysis: $* P<0.05$ in comparison with GKGB; ${ }^{*} P<0.05$ in comparison with GK (all subgroups); ${ }^{\#} P<0.05$ in comparison with GKSS, GKSG, and GKGB; ${ }^{\dagger} P<0.05$ in comparison with GKSG, and GKGB; ${ }^{\#} P<0.05$ in comparison with all other

in comparison with WIC, GKC, and GKSS for GKSG; $P<0.05$ in comparison with GKC, GKSS for GKGB). In all control groups, a slight and not statistically significant increase in plasma glucagon level was observed at $30 \mathrm{~min}$,

groups; ${ }^{\ddagger} P<0.05$ in comparison with WIC, GKC, and GKSG; $P<0.05$ in comparison with GKSS and GKGB; ${ }^{\S} P<0.05$ in comparison with WIC and GKC; ${ }^{\dagger \dagger} P<0.05$ in comparison with WIC, GKSG, and GKGB; ${ }^{\ddagger \pm} P<0.05$ in comparison with GKC and GKSS; ${ }^{\S} P<0.05$ in comparison with WIC, GKC, and GKSS. ${ }^{\mathrm{a}} \boldsymbol{P}<0.05$ in comparison with baseline and ${ }^{\mathrm{b}} \boldsymbol{P}<0.05$ in comparison with previous time point.

without any further change (Fig. 3). Total glucagon secretion during the postprandial period described as AUC was significantly higher in GKSG $(29.9 \pm 0.8 \mathrm{~h} \times$ $\mathrm{ng} / \mathrm{ml})$ and GKGB $(31.5 \pm 1.7 \mathrm{~h} \times \mathrm{ng} / \mathrm{ml})$ in comparison 

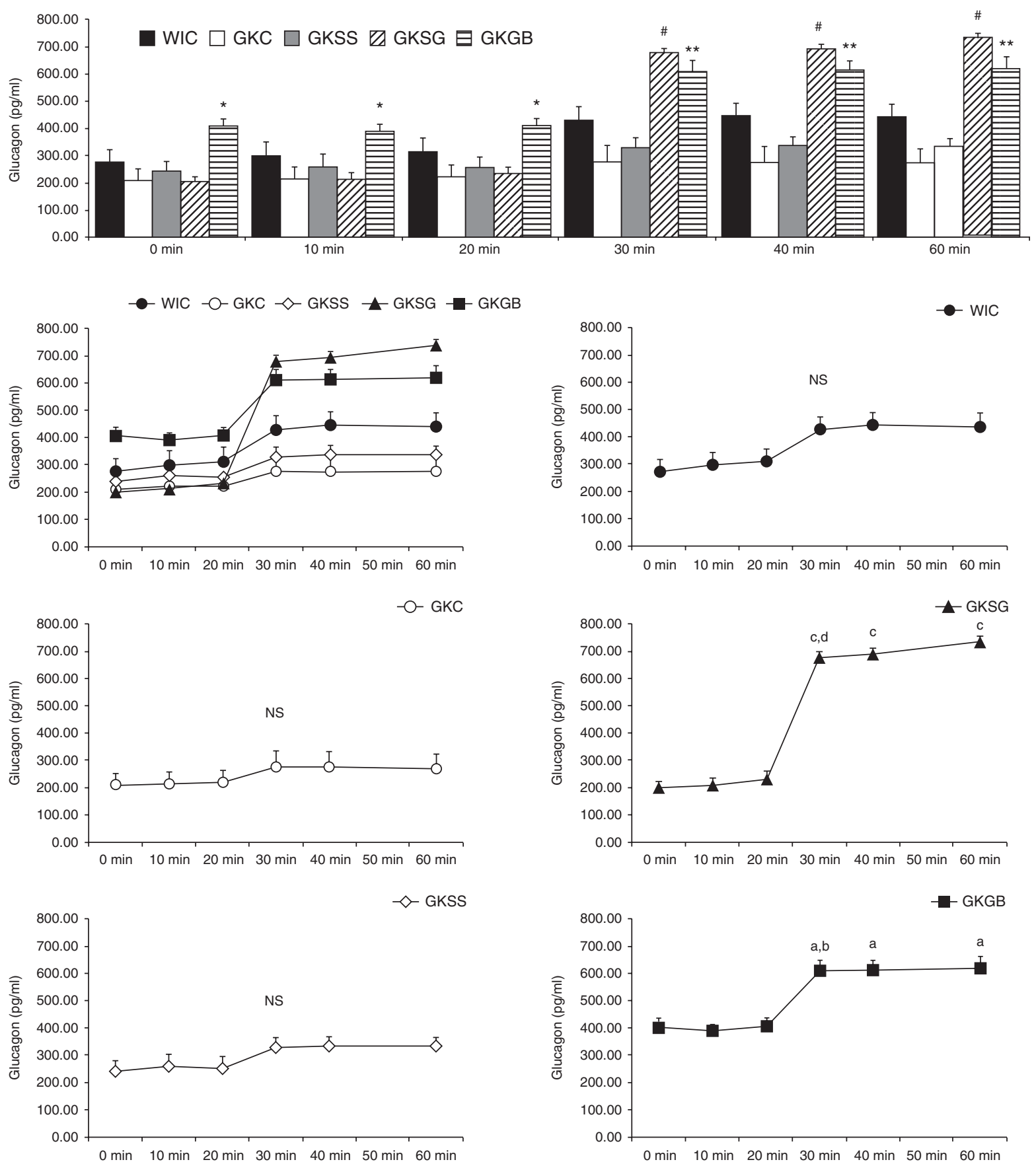

Figure 3

Glucagon level - meal-induced response. WIC, Wistar rats - control; GKC, Goto-Kakizaki rats - control; GKSS, Goto-Kakizaki rats - sham surgery; GKSG, Goto-Kakizaki rats - sleeve gastrectomy; GKGB, Goto-Kakizaki rats - gastric bypass. Kruskal-Wallis test with post hoc analysis: ${ }^{*} P<0.05$ in comparison with GKC and GKSG; ${ }^{~} P<0.05$ in comparison with WIC, GKC,

with GKC $(15.0 \pm 3.0 \mathrm{~h} \times \mathrm{ng} / \mathrm{ml})$ and GKSS $(18.1 \pm 2.0 \mathrm{~h} \times$ $\mathrm{ng} \mathrm{m} / \mathrm{ml}$ ) rats (Table 1 ).

Calculation of the glucagon:insulin ratio (GIR) revealed higher values for GKGB in comparison with

and GKSS; $* * P<0.05$ in comparison with GKC and GKSS. ${ }^{a} P<0.05$ in comparison with baseline; ${ }^{b} P<0.05$ in comparison with previous time point; ${ }^{c} P<0.01$ in comparison with baseline; and ${ }^{d} P<0.01$ in comparison with previous time point.

GKC and GKSS at baseline $(P<0.05)$ and with GKC, GKSS, and GKSG $(P<0.05)$ at $10 \mathrm{~min}$ after the test meal. However, a significant decrease in GIR occurred at 20 min $(P<0.05)$. At this time point, WIC maintained a 
stable GIR $\left(1.0^{-4} \pm 1.68^{-5}\right)$ in comparison with previous time points, significantly higher than GIR in GK rats (all groups, $P<0.05$ ). However, at all following time points up to 60 min GKGB showed an increased GIR in comparison with all control groups $(P<0.05)$. GK rats submitted to sleeve gastrectomy showed a significant increase in GIR at $60 \min \left(1.26^{-4} \pm 6.1^{-6}\right)$, achieving a ratio similar to GKGB rats, and higher than other groups $(P<0.05$ in comparison with WIC, GKC, and GKSS) (Fig. 4).

\section{Discussion}

In our lean animal model of type 2 diabetes, overall glycemic control assessed by HbA1c levels improved after sleeve gastrectomy and gastric bypass. However, mealinduced insulin secretion diminished after surgery, particularly in rats submitted to gastric bypass. The reduction in insulin secretion was accompanied by increased insulin sensitivity. Glugacon secretion was significantly augmented by surgical procedures, in comparison with both GKC and GKSS rats, as well as non-diabetic Wistar rats. In GKGB the GIR started to increase $30 \mathrm{~min}$ after the test meal and remained high throughout the observation period. GKSG rats showed a similar high GIR at $60 \mathrm{~min}$.

Commonalities in $\beta$-cell dysfunction regarding human type 2 diabetes and GK rats include reduction in $\beta$-cell mass, alterations in islets microenvironment, and multiple functional deficits (Seiça et al. 2004, Portha et al. 2009). As expected, GK rats in this study showed impaired metabolic control in comparison with Wistar rats, with significantly worse HbA1c levels and higher blood glucose levels, $2 \mathrm{~h}$ after an OGTT, both at the beginning and at the end of the observation period. However, rats submitted to sleeve gastrectomy or gastric bypass improved their HbA1c levels significantly, although meal-induced insulin secretion (expressed as AUC) did not change (GKSG) or even diminished (GKGB). A similar reduction in insulin secretion has been observed after gastro-jejunal bypass (Weng et al. 2013). Fasting insulin levels do not seem to be affected by surgery (Donglei et al. 2012). Nevertheless, other studies reported conflicting results after a prolonged follow-up (Speck et al. 2011, Trung et al. 2013). Yet, the improved insulin sensitivity observed in our study might have contributed to the amelioration of glucose metabolism.

In general, gastrointestinal surgical procedures such as sleeve gastrectomy and gastric bypass improve diabetes control and represent an accepted therapeutic option, at least in obese patients (Dixon et al. 2011, American
Diabetes Association 2014). They have a well-documented effect on the secretion of gut hormones that, by themselves, are believed to exert a favorable influence on glucose homeostasis. In diabetic patients, the subcutaneous administration of GLP1 analogs improves glycemic control, supposedly through stimulation of glucose-dependent insulin secretion and suppression of glucagon secretion (Shyangdan et al. 2010). As shown in a previous study, meal-induced secretion of GLP1 is enhanced after surgery in GK rats and becomes similar to that of non-diabetic Wistar rats (Eickhoff et al. 2014). However, in this study on pancreatic hormone secretion, improvement of glucose homeostasis was not associated with augmented meal-induced insulin secretion. Furthermore, a study on the effect of sleeve gastrectomy on weight loss and glucose tolerance in GLP1 receptordeficient mice and WT controls showed that postprocedural improvement was similar in the two groups (Wilson-Pérez et al. 2013). Consequently, factors other than direct stimulation of insulin secretion through surgically enhanced GLP1 plasma levels are possibly involved in ameliorated glycemic control (Vidal \& Jiménez 2013).

Glucagon secreted from pancreatic $\alpha$-cells acts as a counter-regulatory hormone for insulin and promotes elevation of blood glucose by hepatic glycogenolysis and gluconeogenesis (Jiang \& Zhang 2003), and a role in the pathophysiology of type 2 diabetes has been proposed (Unger \& Orci 1975, Unger 1985, Quesada et al. 2008). Besides secretion in response to low plasma glucose levels, plasma concentration of glucagon is modulated by insulin at an intra-islet level inhibiting secretion at high insulin levels (Bansal \& Wang 2008). Interestingly, the results of our study showed stable levels of plasma glucagon during the postprandial period with a slight rise at 30 min in WIC, GKC, and GKSS rats, following a significant increase in plasma insulin at $20 \mathrm{~min}$ in GK rats (at $30 \mathrm{~min}$ in WIC). Apparently, no inhibition of glucagon secretion occurred. In rats submitted to sleeve gastrectomy or gastric bypass, a significant increase in plasma glucagon followed a rise in plasma insulin, but with a descending GIR that recovered at $60 \mathrm{~min}$. Although an increased fasting plasma glucagon and delayed glucose-stimulated suppression has been described in humans with type 2 diabetes (Knop et al. 2012), meal-induced or glucose-stimulated glucagon secretion increased after gastric bypass, albeit with improved glucose tolerance (Laferrère et al. 2008, Salehi et al. 2011). A similar postprandial rise in glucagon has been observed in humans with type 1 diabetes, not submitted to surgery, whereas glucagon in $\beta$-cell-competent individuals

Published by Bioscientifica Ltd. 

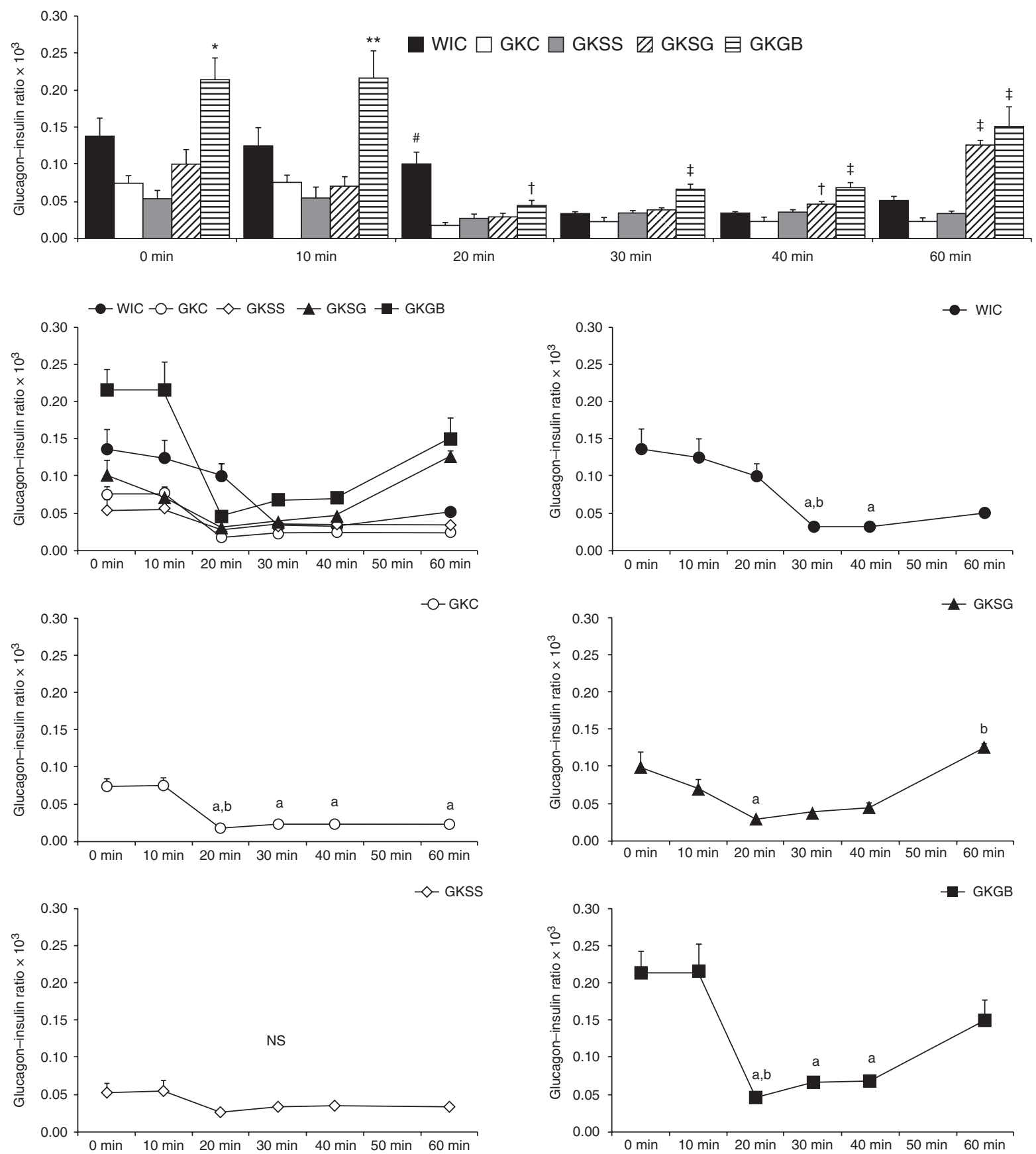

Figure 4

Glucagon-insulin ratio - meal-induced response. WIC, Wistar rats - control; GKC, Goto-Kakizaki rats - control; GKSS, Goto-Kakizaki rats - sham surgery; GKSG, Goto-Kakizaki rats - sleeve gastrectomy; GKGB, Goto-Kakizaki rats - gastric bypass. Kruskal-Wallis test with post hoc analysis: ${ }^{*} P<0.05$ in comparison with GKC and GKSS; $* * P<0.05$ in comparison with GKC, GKSS,

remained stable (Cooperberg \& Cryer 2009). Furthermore, patients submitted to truncal vagotomy with pyloroplasty displayed augmented postprandial plasma glucagon that was not suppressed by the simultaneous administration of

and GKSG; ${ }^{\#} P<0.05$ in comparison with GK (all subgroups); ${ }^{\dagger} P<0.05$ in comparison with GKC; and ${ }^{\ddagger} P<0.05$ in comparison with WIC, GKC, and GKSS. ${ }^{\mathrm{a}} P<0.05$ in comparison to baseline and ${ }^{\mathrm{b}} P<0.05$ in comparison to previous time point.

exogenous GLP1, possibly due impaired vagal innervation (Plamboeck et al. 2013). These observations underscore the importance of other stimuli for the regulation of glucagon secretion besides hypoglycemia, decrease in insulin 
secretion during hypoglycemia, or direct influence of gut hormones.

Impaired pathways with respect to the inhibitory effect of hyperglycemia on glucagon secretion, referred to as $\alpha$-cell resistance, have been identified in studies of $\alpha$-cell lines (Xu et al. 2006). Interestingly, a switch-off signal from zinc atoms, whether bound to insulin or not, also seems to be involved in the initiation of glucagon release from the $\alpha$-cell, as could be shown in streptozotocin-induced diabetic Wistar rats (Zhou et al. 2007). The removal of somatostatinmediated inhibition of glucagon secretion via pancreatic $\delta$-cells by sympathetic activation may further contribute to inadequate glucagon secretion (Hauge-Evans et al. 2010). As indicated by the present data, the multifactorial regulation of glucagon secretion and its importance with respect to glucose metabolism need to be further elucidated.

The results of our study refer to meal-induced plasma levels of insulin and glucagon with respect to a specific type of liquid diet and results may vary with carbohydrate content (Gutniak et al. 1986). Analysis of glucose metabolism was carried out rather early after surgery and might not reflect changes after a more prolonged followup. As such, data should be interpreted with caution with respect to the long-term impact of bariatric and metabolic surgical procedures on plasma glucagon. However, our results call into question the role of hyperglucagonemia in glucose intolerance in a lean animal model of type 2 diabetes, indicating that the role of glucagon in glucose metabolism after bariatric and metabolic surgery requires further study.

\section{Conclusion}

Lean diabetic GK rats showed similar glucagon levels in comparison with non-diabetic Wistar rats, without any significant modification in response to a mixed meal. GK rats submitted to sleeve gastrectomy or gastric bypass showed a significant rise in glucagon, $30 \mathrm{~min}$ after a mixed meal, as well as an increased GIR at $60 \mathrm{~min}$, albeit with a significant amelioration of overall glycemic control. In GK rats submitted to sleeve gastrectomy or gastric bypass, enhanced post-procedural glucagon secretion did not interfere with improved glucose tolerance. The role of glucagon in glycemic control after bariatric and metabolic surgery deserves further investigation.

\section{Declaration of interest}

The authors declare that there is no conflict of interest that could be perceived as prejudicing the impartiality of the research reported.

\section{Funding}

This research did not receive any specific grant from any funding agency in the public, commercial or not-for-profit sector.

\section{Acknowledgements}

All research was done at the Laboratory for Experimental Research, the Institute of Physiology, and the Institute for Biomedical Imaging and Life Sciences (IBILI), Faculty of Medicine, University of Coimbra, Coimbra, Portugal.

\section{References}

American Diabetes Association 2014 Standards of medical care in diabetes2014. Diabetes Care 37(Suppl 1) S14-S80. (doi:10.2337/dc14-S014)

Bansal P \& Wang Q 2008 Insulin as a physiological modulator of glucagon secretion. American Journal of Physiology. Endocrinology and Metabolism 295 E751-E761. (doi:10.1152/ajpendo.90295.2008)

de Bona Castelan J, Bettiol J, d'Acampora AJ, Castelan JVE, de Souza JC, Bressiani V \& Giroldi SB 2007 Sleeve gastrectomy model in Wistar rats. Obesity Surgery 17 957-961. (doi:10.1007/s11695-007-9150-y)

Buchwald H, Estok R, Fahrbach K, Banel D, Jensen MD, Pories WJ, Bantle JP \& Sledge I 2009 Weight and type 2 diabetes after bariatric surgery: systematic review and meta-analysis. American Journal of Medicine 122 248-256.e5. (doi:10.1016/j.amjmed.2008.09.041)

Cooperberg BA \& Cryer PE 2009 -cell-mediated signaling predominates over direct $\alpha$-cell signaling in the regulation of glucagon secretion in humans. Diabetes Care 32 2275-2280. (doi:10.2337/dc09-0798)

DeFronzo RA 2009 Banting Lecture. From the triumvirate to the ominous octet: a new paradigm for the treatment of type 2 diabetes mellitus. Diabetes 58 773-795. (doi:10.2337/db09-9028)

Dixon JB, Zimmet P, Alberti KG \& Rubino F 2011 Bariatric surgery: an IDF statement for obese Type 2 diabetes. Diabetic Medicine 28 628-642. (doi:10.1111/j.1464-5491.2011.03306.x)

Donglei Z, Liesheng L, Xun J, Chenzhu Z \& Weixing D 2012 Effects and mechanism of duodenal-jejunal bypass and sleeve gastrectomy on GLUT2 and glucokinase in diabetic Goto-Kakizaki rats. European Journal of Medical Research 17 15. (doi:10.1186/2047-783X-17-15)

Eickhoff H, Louro TM, Matafome PN, Vasconcelos F, Seiça RM \& Castro e Sousa F 2014 Amelioration of glycemic control by sleeve gastrectomy and gastric bypass in a lean animal model of type 2 diabetes: restoration of gut hormone profile. Obesity Surgery (In Press). (doi:10.1007/s11695014-1309-8)

Gastaldelli A, Baldi S, Pettiti M, Toschi E, Camastra S, Natali A, Landau BR \& Ferrannini E 2000 Influence of obesity and type 2 diabetes on gluconeogenesis and glucose output in humans: a quantitative study. Diabetes 49 1367-1373. (doi:10.2337/diabetes.49.8.1367)

Gutniak M, Grill V \& Efendić S 1986 Effect of composition of mixed meals-low- versus high-carbohydrate content-on insulin, glucagon, and somatostatin release in healthy humans and in patients with NIDDM. Diabetes Care 9 244-249. (doi:10.2337/diacare.9.3.244)

Hauge-Evans AC, King AJ, Fairhall K, Persaud SJ \& Jones PM 2010 A role for islet somatostatin in mediating sympathetic regulation of glucagon secretion. Islets 2 341-344. (doi:10.4161/isl.2.6.13858)

Holst JJ, Vilsbøll T \& Deacon CF 2009 The incretin system and its role in type 2 diabetes mellitus. Molecular and Cellular Endocrinology 297 127-136. (doi:10.1016/j.mce.2008.08.012)

Jiang G \& Zhang BB 2003 Glucagon and regulation of glucose metabolism. American Journal of Physiology. Endocrinology and Metabolism 284 E671-E678. (doi:10.1152/ajpendo.00492.2002)

Katz A, Nambi SS, Mather K, Baron AD, Follmann DA, Sullivan G \& Quon MJ 2000 Quantitative insulin sensitivity check index: a simple, accurate method for assessing insulin sensitivity in humans. Journal of 
Clinical Endocrinology and Metabolism 85 2402-2410. (doi:10.1210/ jcem.85.7.6661)

Kilkenny C, Browne WJ, Cuthill IC, Emerson M \& Altman DG 2010 Improving bioscience research reporting: the ARRIVE guidelines for reporting animal research. PLoS Biology 8 e1000412. (doi:10.1371/ journal.pbio.1000412)

Knop FK, Aaboe K, Vilsbøll T, Vølund A, Holst JJ, Krarup T \& Madsbad S 2012 Impaired incretin effect and fasting hyperglucagonaemia characterizing type 2 diabetic subjects are early signs of dysmetabolism in obesity. Diabetes, Obesity \& Metabolism 14 500-510. (doi:10.1111/ j.1463-1326.2011.01549.x)

Laferrère B, Teixeira J, McGinty J, Tran H, Egger JR, Colarusso A, Kovack B, Bawa B, Koshy N, Lee H et al. 2008 Effect of weight loss by gastric bypass surgery versus hypocaloric diet on glucose and incretin levels in patients with type 2 diabetes. Journal of Clinical Endocrinology and Metabolism 93 2479-2485. (doi:10.1210/jc.2007-2851)

Meguid MM, Ramos EJB, Suzuki S, Xu Y, George ZM, Das UN, Hughes K, Quinn R, Chen C, Marx W et al. 2004 A surgical rat model of human Roux-en-Y gastric bypass. Journal of Gastrointestinal Surgery 8 621-630. (doi:10.1016/j.gassur.2004.02.003)

Muniyappa R, Chen H, Muzumdar R, Einstein FH, Yan X, Yue LQ, Barzilai N \& Quon MJ 2009 Comparison between surrogate indexes of insulin sensitivity/resistance and hyperinsulinemic euglycemic clamp estimates in rats. American Journal of Physiology. Endocrinology and Metabolism 297 E1023-E1029. (doi:10.1152/ajpendo.00397.2009)

Papamargaritis D, le Roux CW, Sioka E, Koukoulis G, Tzovaras G \& Zacharoulis D 2013 Changes in gut hormone profile and glucose homeostasis after laparoscopic sleeve gastrectomy. Surgery for Obesity and Related Diseases 9 192-201. (doi:10.1016/j.soard.2012.08.007)

Plamboeck A, Veedfald S, Deacon CF, Hartmann B, Wettergren A, Svendsen LB, Meisner S, Hovendal C, Vilsbøll T, Knop FK et al. 2013 The effect of exogenous GLP-1 on food intake is lost in male truncally vagotomized subjects with pyloroplasty. American Journal of Physiology. Gastrointestinal and Liver Physiology 304 G1117-G1127. (doi:10.1152/ ajpgi.00035.2013)

Portha B, Lacraz G, Kergoat M, Homo-Delarche F, Giroix M-H, Bailbé D, Gangnerau M-N, Dolz M, Tourrel-Cuzin C \& Movassat J 2009 The GK rat $\beta$-cell: a prototype for the diseased human $\beta$-cell in type 2 diabetes? Molecular and Cellular Endocrinology 297 73-85. (doi:10.1016/j.mce. 2008.06.013)

Quesada I, Tudurí E, Ripoll C \& Nadal A 2008 Physiology of the pancreatic $\alpha$-cell and glucagon secretion: role in glucose homeostasis and diabetes. Journal of Endocrinology 199 5-19. (doi:10.1677/JOE-08-0290)

Salehi M, Prigeon RL \& D'Alessio DA 2011 Gastric bypass surgery enhances glucagon-like peptide 1-stimulated postprandial insulin secretion in humans. Diabetes 60 2308-2314. (doi:10.2337/db11-0203)

Scopinaro N, Adami GF, Papadia FS, Camerini G, Carlini F, Briatore L, D'Alessandro G, Parodi C, Weiss A, Andraghetti G et al. 2011 The effects of biliopancreatic diversion on type 2 diabetes mellitus in patients with mild obesity (BMI 30-35 kg/m²) and simple overweight (BMI $25-30 \mathrm{~kg} / \mathrm{m}^{2}$ ): a prospective controlled study. Obesity Surgery 21 880-888. (doi:10.1007/s11695-011-0407-0)

Seiça RM, Martins MJ, Pessa PB, Santos RM, Do Rosário LM, Suzuki KI \& Martins MI 2003 [Morphological changes of islet of Langerhans in an animal model of type 2 diabetes]. Acta Médica Portuguesa 16 381-388.
Seiça RM, Suzuki KI, Santos RM \& Do Rosário LM 2004 [Impaired insulin secretion in isolated islets of Goto-Kakizaki rats, an animal model of non obese type 2 diabetes, is a primary event]. Acta Médica Portuguesa 17 42-48.

Shukla AP, Moreira M, Dakin G, Pomp A, Brillon D, Sinha N, Strain GW, Lebovitz H \& Rubino F 2012 Medical versus surgical treatment of type 2 diabetes: the search for level 1 evidence. Surgery for Obesity and Related Diseases 8 476-482. (doi:10.1016/j.soard.2012.03.004)

Shyangdan DS, Royle PL, Clar C, Sharma P \& Waugh NR 2010 Glucagon-like peptide analogues for type 2 diabetes mellitus: systematic review and meta-analysis. BMC Endocrine Disorders 1020. (doi:10.1186/1472-6823-10-20)

Speck M, Cho YM, Asadi A, Rubino F \& Kieffer TJ 2011 Duodenal-jejunal bypass protects GK rats from $\beta$-cell loss and aggravation of hyperglycemia and increases enteroendocrine cells coexpressing GIP and GLP-1. American Journal of Physiology. Endocrinology and Metabolism 300 E923-E932. (doi:10.1152/ajpendo.00422.2010)

Thaler JP \& Cummings DE 2009 Minireview: Hormonal and metabolic mechanisms of diabetes remission after gastrointestinal surgery. Endocrinology 150 2518-2525. (doi:10.1210/en.2009-0367)

Trung VN, Yamamoto H, Yamaguchi T, Murata S, Akabori H, Ugi S, Maegawa H \& Tani T 2013 Effect of sleeve gastrectomy on body weight, food intake, glucose tolerance, and metabolic hormone level in two different rat models: Goto-Kakizaki and diet-induced obese rat. Journal of Surgical Research 185 159-165. (doi:10.1016/j.jss.2013. 05.019)

Unger RH 1985 Glucagon physiology and pathophysiology in the light of new advances. Diabetologia 28 574-578. (doi:10.1007/ BF00281991)

Unger RH \& Orci L 1975 The essential role of glucagon in the pathogenesis of diabetes mellitus. Lancet 1 14-16. (doi:10.1016/S01406736(75)92375-2)

Vidal J \& Jiménez A 2013 Diabetes remission following metabolic surgery: is GLP-1 the culprit? Current Atherosclerosis Reports 15357. (doi:10.1007/s11883-013-0357-1)

Weng S-G, Zhang B, Feng S, Xu C-G \& Lin C-Z 2013 Effects of modified biliopancreatic diversion on glucose tolerance of GK rats. Obesity Surgery 23 522-530. (doi:10.1007/s11695-012-0830-x)

Wilson-Pérez HE, Chambers AP, Ryan KK, Li B, Sandoval DA, Stoffers D, Drucker DJ, Pérez-Tilve D \& Seeley RJ 2013 Vertical sleeve gastrectomy is effective in two genetic mouse models of glucagon-like Peptide 1 receptor deficiency. Diabetes 62 2380-2385. (doi:10.2337/db12-1498)

Xu Y, Ohinata K, Meguid MM, Marx W, Tada T, Chen C, Quinn R \& Inui A 2002 Gastric bypass model in the obese rat to study metabolic mechanisms of weight loss. Journal of Surgical Research 107 56-63. (doi:10.1016/S0022-4804(02)96508-X)

Xu E, Kumar M, Zhang Y, Ju W, Obata T, Zhang N, Liu S, Wendt A, Deng S, Ebina $Y$ et al. 2006 Intra-islet insulin suppresses glucagon release via

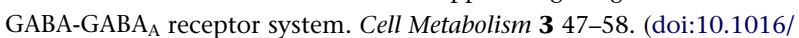
j.cmet.2005.11.015)

Zhou H, Zhang T, Harmon JS, Bryan J \& Robertson RP 2007 Zinc, not insulin, regulates the rat $\alpha$-cell response to hypoglycemia in vivo. Diabetes 56 1107-1112. (doi:10.2337/db06-1454)

Received in final form 24 September 2014

Accepted 1 October 2014

Accepted Preprint published online 1 October 2014 http://joe.endocrinology-journals.org DOI: $10.1530 / \mathrm{JOE}-14-0445$
(C) 2014 Society for Endocrinology Printed in Great Britain
Published by Bioscientifica Ltd 